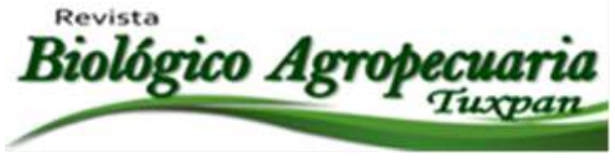

\title{
Causas asociadas a la reprobación escolar en estudiantes de enfermería.
}

\author{
Causes associated with school failure in nursing students
}

\author{
Nazaria Martínez Díaz ${ }^{1 凶}$, Laura Elena Urbina Sánchez ${ }^{1}$, David Zepeta Hernández ${ }^{1}$, Higinio Fernández Sánchez ${ }^{1}$ \\ y Erika Mayte Del Ángel Salazar ${ }^{1}$ \\ ${ }^{1}$ Facultad de Enfermería de la Universidad Veracruzana Región Poza Rica-Tuxpan, México. \\ Autor para correspondencia: nmartinez@uv.mx
}

Recibido: $15 / 07 / 2017$

Aceptado: 10/12/2017

\section{RESUMEN}

Introducción: Reprobación escolar es el resultado de un proceso que detiene, limita o no acredita el avance del alumno en su vida académica. La reprobación constituye una dificultad de los estudiantes para adquirir los conocimientos, habilidades y aptitudes que se concreta con el bajo rendimiento académico, la reprobación y en su forma extrema en la deserción. Objetivo: Identificar las causas de reprobación de los estudiantes de Licenciatura en Enfermería de una Facultad de la Universidad Veracruzana. Métodos: Estudio descriptivo, transversal con muestreo no probabilístico por conveniencia, con 93 participantes. Se aplicó el instrumento causas de reprobación en universitarios con 24 ítems, tres dimensiones, la primera atribuible a los estudiantes, la segunda a los docentes y la tercera a la situación socioeconómica. Resultados: La edad promedio fue de 22 años, predominando el sexo de las mujeres. El 55\% respondió que reprueba por no organizar su tiempo para estudiar y realizan tareas cuando están presionados, el $46 \%$ atribuye reprobar por falta de retroalimentación, el $44 \%$ a la relación maestro-alumno. El 27\% refiere reprobar por el uso de las redes sociales y el $24 \%$ porque tiene que trabajar para pagar sus estudios y sostener a su familia. Conclusiones: Las causas de reprobación de los estudiantes de enfermería son por no organizar el tiempo para estudiar y hacer tareas, falta de retroalimentación y relación maestro-alumno, trabajar para estudiar y sostener la familia.

Palabras claves: Reprobación escolar, Educación superior, Enfermería.

\section{ABSTRAC}

Background: School failure is the result of a process that stops, limits or does not accredit the progress of the student in his academic life. Student failure is a general phenomenon also known as "school failure", it's described as the difficulty that some students have during the acquisition of knowledge, skills and aptitudes taught within the school itself, which materializes in low academic performance, student failure and in its extreme form in desertion. Aim: To identify the causes of student failure of undergraduate nursing students of the University of Veracruz. Method: The study design was 
quantitative, at a descriptive level, non-probabilistic sampling of 93 participants. The instrument used was "causes of student failure in college students", which consisted of 35 items divided into three dimensions; the first attributable to students, the second to teachers and the third to the socioeconomic situation of the student. Results: The average age of participants is 22 years, mainly female participants. According to the causes attributable to students, $55 \%$ responded that they fail to organize their study time and to perform tasks when they are put under pressured. As for the causes inherent to the teacher, $46 \%$ say if to the lack of feedback, and $44 \%$ due do to the teacher-student relationship. Finally, concerning the socioeconomic dimension, $27 \%$ refer to the use of social networks as a cause of student failure, while $24 \%$ said they fail because they have to work in order to pay for their education and support their family. Conclusion: The authors conclude, the main causes of student failure among nursing students are for not being able to organize their study time, lack of teacher feedback and to have a job in order to support their family.

Keywords: College failure, Higher education, Nursing

\section{INTRODUCCIÓN}

La educación superior en México enfrenta múltiples situaciones problemáticas, entre ellas el elevado índice de reprobación, la cuales se considera necesario atender con la inmediatez que el caso requiere, ya que lo contrario, favorece el decremento del bienestar público. Por otra parte, la reprobación escolar, tiene una profunda connotación negativa, de descalificación, de reproche, de censura, de condena y de desaprobación, por lo que no queda duda de que afecta en gran medida la dignidad del alumno identificado como "reprobado" (Márquez, 2007).

Alcanzar el rendimiento académico en la educación requiere del análisis de los procedimientos y de los factores que afectan el proceso enseñanza aprendizaje. Habitualmente se ha contemplado que el problema se divide en varios factores, uno de ellos, al que más se evalúa, es el estudiante y se le señala como el más responsable del problema de reprobación (Flores, Camacho y Ontiveros, 2013).

Actualmente el sistema de educación superior mexicano otorga cobertura al $29.4 \%$ de la población de 19-23 años. La educación superior se distribuye en un $3.9 \%$ de estudiantes de educación técnica superior, $88.9 \%$ de licenciatura, y 7.2 de estudiantes de posgrado, como se puede observar el nivel más significativo es el de licenciatura (Aguirre, 2014).

Jiménez (2000) entiende como rendimiento escolar el nivel de conocimientos demostrados en un área o materia comparado con la norma de edad y nivel académico. Debido a que es cuantificable, dicho rendimiento puede medirse puesto que acepta valores medibles entre 0 a 10 o en su caso de 0 a 100, podemos en un momento dado determinar el éxito o fracaso de un alumno. En este mismo sentido, gran parte de la responsabilidad en cuanto al rendimiento del alumno la tiene el maestro, así como el proceso de enseñanza aprendizaje, la metodología del profesor, el alumno en sí, el apoyo familiar, la situación social. Se entiende que el rendimiento no son solo las calificaciones del estudiante, sino también sus habilidades, destrezas, aptitudes, ideales e intereses entre otros (Figueroa, 2004). Es por esto que la reprobación constituye un elemento de un fenómeno más general denominado "fracaso escolar ". Dicho fenómeno ha recibido una creciente atención en años recientes, entendiéndose como la dificultad que manifiestan algunos alumnos para adquirir los

Revista Científica Biológico Agropecuaria Tuxpan 5 (2) 
conocimientos, habilidades y aptitudes que se enseñan en la escuela misma que se concretiza en el bajo rendimiento académico, la reprobación $\mathrm{y}$, en su forma extrema, en la deserción (Martínez, 2010).

El abandono de los estudios, la reprobación y el rezago constituyen elementos imbricados en un fenómeno educativo más amplio adjetivado como fracaso escolar, que altera de manera muy importante las trayectorias estudiantiles de los individuos e incluso, la vida en general de los afectados. En años recientes, estos temas han adquirido relevancia en la educación de nivel superior, tanto en su diagnóstico como en el diseño de las posibles estrategias educativas requeridas para su solución (Flores, Camacho y Ontiveros, 2013). Entre los principales factores que influyen en la reprobación estudiantil se consideran aspectos económicos, políticos, sociales, familiares, psicológicos y pedagógicos, entre otros (INEE, 2008). Referente al aspecto económico, se incluyen la falta de recursos en el hogar para enfrentar los gastos que demandan la asistencia a la escuela, así como la necesidad de trabajar o buscar empleo. De igual manera, se deben de considerar las expectativas familiares, su composición, su forma y estilo de vida (Abril et al., 2008). Los factores físicos pueden impedir que los alumno presenten un buen proceso de enseñanza-aprendizaje debido a que estos factores le puedan impedir escuchar, aprender, leer, y observar correctamente la explicación que da el maestro durante su clase, teniendo como consecuencia las bajas calificaciones, las cuales no son por no poseer capacidad, sino por el impedimento físico. El cierto grado de cansancio puede obstaculizar su correcto rendimiento escolar, también está comprobado que aquellos jóvenes que duermen poco o mal, y los que no son alimentados correctamente, especialmente en el desayuno rinden menos de lo deseable (Carrasco, 2011).

La relación existente entre el alumno y el maestro es un factor básico en la determinación del clima general del salón de clases. Por lo tanto es importante determinar las diferentes bases de relación ya que tanto maestros como alumnos lo perciben; existiendo estos factores va a ocasionar que el alumno no sienta deseos de aprender, la necesidad de logro, el interés por un futuro campo laboral, sobresalir e incluso el reconocimiento de sus padres y maestros. Cuando estas motivaciones se ven afectadas por diversas circunstancias las actividades del alumno disminuyen en calidad o dejan de asistir a clases, no cumplen con tareas o simplemente ya no tienen interés por seguir adelante. Todo esto se refleja en el desempeño escolar que puede llevar a la reprobación (Aburto, 2010). Evidentemente no podemos desconocer que la reprobación y deserción escolar son fenómenos psicosociales complejos, en los que participan factores estructurales, sociales, familiares e individuales y que tienen consecuencias en igual número de niveles de la realidad desde el sistema educativo hasta la autoestima de los individuos. Por ello, resulta un grave error atribuirlos solo a causas personales, como la falta de dedicación, la calidad académica o el compromiso con la escuela por parte de los alumnos, y suponer que el asunto debe resolverse en ese nivel, es decir de manera individual (Nava, 2007).

Ante este panorama, el objetivo del presente estudio fue identificar las causas de reprobación en estudiantes de una Facultad de Enfermería de la zona norte del Estado de Veracruz.

\section{MATERIALES Y MÉTODOS}

Estudio descriptivo y transversal. La población se conformó por estudiantes de enfermería que habían reprobado alguna experiencia educativa, la muestra estuvo constituida por 93 estudiantes de ambos sexos, inscritos en el periodo agosto 2016-enero 2017. El muestreo fue no probabilístico por conveniencia y la recolección de datos fue en el periodo febrero a marzo de 2017.

Los criterios de selección, se incluyeron aquellos estudiantes que aceptaron participar voluntariamente y que tuvieran antecedentes de reprobación escolar universitaria, no se 
excluyeron instrumentos ya que fueron contestados acorde a las instrucciones indicadas por los investigadores. La encuesta utilizada fue el cuestionario denominado "Causas de reprobación universitaria" elaborado por Martínez-Díaz, Urbina-Sánchez, ZepetaHernández y Fernández-Sánchez en el año 2016, académicos de la Facultad de Enfermería de la Universidad Veracruzana, Región Poza RicaTuxpan. El cuestionario está conformado por 24 ítems con respuestas dicotómicas de "si" o "no" y está estructurado en 3 dimensiones: factores inherentes al estudiante del ítem 1 al 8, factores inherentes al académico del ítem 9 al 14 y factores inherentes al nivel socio-económico del ítem 15 al 24; la escala de evaluación es de 1 a 2 y se presenta el factor más representativo para los participantes en términos porcentuales. Los datos obtenidos de la investigación fueron tabulados en el programa SPSS versión 22.0, haciendo uso de la estadística descriptiva; frecuencia, porcentaje para describir los factores asociados a la reprobación universitaria y el perfil de los estudiantes de enfermería.

El estudio se apegó a las disposiciones generales de la Ley General de Salud en materia de investigación para la salud

(Secretaria de Salud, 2012) específicamente en los apartados que se refieren a la garantía social de derecho y a la protección de salud en materia de investigación, y bajo estricto consentimiento informado de los participantes.

\section{RESULTADOS}

Se encuestó a 93 estudiantes con antecedentes de reprobación escolar universitaria, con una edad promedio de $22 \pm 3.67$ años (IC95 21.24-22.76), una edad mínima de 18 y máxima de 49 años predominando el sexo mujer con un $71 \%$ y estado civil soltero con un $88.2 \%$ (Cuadro I).

Las experiencias educativas que presentan mayor índice de reprobación son inglés II, anatomía y fisiología, enfermería en la atención comunitaria, bioestadística y ética y legislación, principalmente. Con respecto al número de experiencias educativas que reprueban los estudiantes por semestre, se encontró que el $73 \%$ reprueba una experiencia educativa, el 19\% reprueba dos experiencias educativas, el $7 \%$ tres y el $1 \%$ cinco experiencias educativas. Dentro de las causas de reprobación atribuibles a los estudiantes, el 55\% respondió que dentro de las principales causas de reprobación son el no organizar sus tiempos para estudiar y realizar tareas escolares y el $41.9 \%$ refirieron estudiar únicamente cuando se sienten presionados por exámenes o trabajos que tienen que entregar.

Cuadro I. Sexo y estado civil de los estudiantes

\begin{tabular}{lccc}
\hline \multicolumn{2}{c}{ Datos sociodemográficos } & Frecuencia & Porcentaje \\
\hline Sexo & Mujer & 66 & 71 \\
\multicolumn{2}{c}{ Estado Civil } & 27 & 29 \\
& Sombre & 82 & 88.2 \\
& Soltero & 5 & 5.4 \\
& Casado & 6 & 6.5 \\
\hline
\end{tabular}

Fuente: Directa

En lo que respecta a las causas de reprobación inherentes al docente, el $46 \%$ refieren que es por la falta de retroalimentación de los temas abordados y tareas solicitadas por el profesor y el $44 \%$ considera que es por la relación 


\section{DISCUSIÓN}

estudiante-maestro. Por último, dentro de las causas de reprobación inherentes a la situación socioeconómica del estudiante, el $27 \%$ refieren que el usar redes sociales como "Facebook", "twitter", "whats app", entre otros son factores atribuibles a la reprobación, además, el 24\% manifestó que considera una causa la necesidad de trabajar para pagar sus estudios y sostener a sus familias (Cuadro II).
Los resultados de la presente investigación sobre causas de reprobación realizada muestra que el $54.8 \%$ de los estudiantes reprueban por no organizar su tiempo para estudiar y realizar tareas escolares, semejante a los resultados de Amado et al. (2014) que reportan que el $80 \%$ de los estudiantes dedican pocas horas al estudio; mientras que Llanes-Castillo et al. (20113) encontró que el $40 \%$ de los estudiantes reprueba por no estudiar lo suficiente y ponerse nervioso al contestar los exámenes.

Cuadro II. Principales causas de reprobación de los estudiantes

\begin{tabular}{lcccc}
\multicolumn{1}{c}{ Causas de reprobación } & \multicolumn{3}{c}{ SI } & \multicolumn{2}{c}{ NO } \\
\cline { 2 - 5 } & f & $\%$ & f & $\%$ \\
\hline $\begin{array}{l}\text { Inherentes al estudiante } \\
\quad \begin{array}{l}\text { Estudiar únicamente cuando me siento } \\
\text { presionado }\end{array}\end{array}$ & 39 & 41.9 & 54 & 58.1 \\
$\begin{array}{l}\text { No organizar mi tiempo para estudiar y } \\
\text { realizar tareas escolares }\end{array}$ & 51 & 54.8 & 42 & 45.2 \\
$\begin{array}{l}\text { No entregar puntualmente las tareas escolares } \\
\text { No tomar apuntes durante la clase }\end{array}$ & 26 & 28 & 67 & 72 \\
$\quad$ No asistir a clases & 19 & 20.4 & 74 & 79.6 \\
El interés en la carrera & 25 & 26.9 & 68 & 73.1 \\
\end{tabular}

\section{Inherentes al docente}

Falta de retroalimentación de los temas

abordados y tareas solicitadas

$\begin{array}{llll}43 & 46.2 & 50 & 53.8 \\ 41 & 44.1 & 52 & 55.9 \\ 33 & 35.5 & 60 & 64.5 \\ 32 & 34.4 & 61 & 65.6 \\ 24 & 25.8 & 69 & 74.2\end{array}$

La relación estudiante-maestro

Falta de explicación del maestro

Los métodos de enseñanza-aprendizaje no son variados

\section{Inherentes a la socio-economía del estudiante}

Trabajar para pagar mis estudios y/o sostener a mi familia

El uso inadecuado de redes sociales (whats app, facebook, twitter).

$\begin{array}{llll}25 & 26.9 & 68 & 73.1\end{array}$




\section{Cuadro II. Principales causas de reprobación de los estudiantes}

\begin{tabular}{|c|c|c|c|c|}
\hline \multirow{2}{*}{ Causas de reprobación } & \multicolumn{2}{|c|}{ SI } & \multicolumn{2}{|c|}{ NO } \\
\hline & $\mathrm{f}$ & $\%$ & $\mathrm{f}$ & $\%$ \\
\hline $\begin{array}{l}\text { No contar con el material necesario para } \\
\text { estudiar }\end{array}$ & 20 & 21.5 & 73 & 78.5 \\
\hline No disponer de beca para estudiante & 19 & 20.4 & 74 & 79.6 \\
\hline $\begin{array}{l}\text { Las relaciones afectivas (novio, amigos, } \\
\text { compañeros, familia). }\end{array}$ & 12 & 12.9 & 81 & 87.1 \\
\hline
\end{tabular}

Fuente: Directa

Respecto a las causas de reprobación inherentes al maestro, en el presente estudio se encontró que el $46.2 \%$ de los estudiantes mencionaron que se debe a la falta de retroalimentación del profesor de los temas abordados y tareas solicitadas y el $44.1 \%$ lo atribuye a la relación maestro alumno, similar a lo reportado por Saucedo et al. (2016) que encontró que el 62\% de sus encuestados reprueban por estas mismas causas señalando que el profesor no explica con claridad, y no proporciona suficiente material didáctico y exámenes que no evalúan los temas vistos.

En relación a las causas socioeconómicas de reprobación, se encontró que el $26.9 \%$ de los estudiantes dijeron haber reprobado por el uso inadecuado de las redes sociales, el $23.7 \%$ de los encuestados menciono reprobar porque tiene que trabajar para pagar sus estudios y sostener a su familia, al respecto, Saucedo et al. (2016) menciona que el $14.8 \%$ de sus encuestados comento haber reprobado porque trabajan y estudian; en tanto que Amado et al. (2014) reportó que el $46 \%$ atribuye haber reprobado por causas sociales y problemas familiares entre los que se encuentran embarazos y cuidado de sus hijos.

\section{CONCLUSIÓN}

La reprobación de los estudiantes universitarios es una situación que preocupa y ocupa a las instituciones de educación superior, los resultados muestran que las causas de reprobación atribuibles a los estudiantes son el estudiar únicamente cuando se sienten presionados (examen, trabajos, exposiciones) y el no organizar el tiempo para estudiar y realizar tareas escolares diariamente.

Entre las causas de reprobación inherentes al maestro predomina el hecho de que se percibe una falta de retroalimentación de los temas abordados y tareas solicitadas y la falta de empatía en la relación estudiante-maestreo en el proceso de enseñanza-aprendizaje.

En cuanto a las causas de reprobación atribuibles a la situación socioeconómica de los estudiantes, se observa que dentro de las principales está el tener que trabajar para pagar los propios estudios y al mismo tiempo sostener a la familia y el uso indiscriminado de las redes sociales (whats app, facebook, twitter), mismas que se convierten en un potencial distractor para la atención a las clases y cumplimiento de las tareas.

\section{LITERATURA CITADA}

Abril, E., Román, R., Cubillas, M. y Moreno, I. (2008) ¿Deserción o autoexclusión? Un análisis de las causas de abandono escolar en estudiantes de educación media superior en Sonora, México [versión electrónica]. Revista Electrónica de Investigación Educativa, 10(1). Disponible en: http://redie.uabc.mx/vol10no1/contenidoabril.html

Aburto, M. (2010). Factores escolares que influyen en la reprobación. Caso facultad de bioanalisis.

Disponible 
en:http://cdigital.uv.mx/bitstream/12345678 9/39705/1/aburtoelizondo.pdf

Aguirre, M. (2014): Educación en México. Carpeta de indicadores y carpetas sociales.

Amado, M. M. G., García, V. Á., Brito, P. R. A., Sánchez, L. B. I., \& Sagaste, B. C. A. (2014). Causas de reprobación en ingeniería desde la perspectiva del académico y administradores.

Carrasco, C. (2011). Factores que influyen en la reprobación en química general II en la Universidad Pedagógica Francisco Morazan. Scribd. Recuperado el 2 de Febrero de 2017, de Scribd: https://es.scribd.com/doc/74653247/Informe -Final-Investigacion-Cualitativa-sobre-laReprobacion-en-Quimica-General-II-en-laUPNFM-Honduras-2011

Figueroa, C. (2004). Sistema de evaluaciones académicas ( $1^{\mathrm{a}}$.Ed.). El Salvador Universitaria. Revista Iberoamericana par la investigación y el desarrollo educativo ISSN 2007/7467

Flores, S., Camacho, A. y Ontiveros, R. (2013) Análisis estadístico de las causas de reprobación desde la perspectiva del alumno de Ingeniería del Instituto Tecnológico de Chihuahua. Disponible en www.chi.itesm.mx/investigacion/wpcontent/uploads/2013/11/EDU42.

INNE. Instituto Nacional para la Evaluación de la Educación. 2008 Panorama Educativo de México. Indicadores del Sistema Educativo Nacional. Disponible en: http://www.inee. edu.mx/index.php/publicaciones/informesin stitucionales/panorama-educativo/3742.
Llanes-Castillo, A, Cervantes-López, M.J., PeñaMaldonado, A. A. y Saldívar- González, A. H. (2013) Factores asociados a la reprobación de los estudiantes de la Licenciatura de Médico Cirujano. Disponible en: http://132.248.9.34/hevila/RevistadelaEscue ladeMedicinaDrJoseSierraFloresdelaUniver sidaddelNoreste/2013/vol27/no1/5.pdf

Márquez, L.R. (2007). Las políticas públicas en relación a la reprobación escolar en la educación media superior: el caso de los subsistemas descentralizados de San Luis Potosí. Tesis, Maestría en Políticas Públicas Comparadas, FLACSO México, México.

Martinez, A. (2013). Factores asociados a la reprobacion estudiantil en la sierra Sur Oaxaca. Oaxca, Mexico.

Martínez, M. (2010). Análisis multirreferencial del fenómeno de la reprobación en estudiantes universitarios mexicanos. Disponible en http://www.scielo.br/scielo.php?script=sci_ arttext\&pid=S1413-85571998000200010

Nava Bustos G (2007) Factores de reprobación en los alumnos del Centro Universitario de Ciencias de la Salud de la Universidad de Guadalajara. Revista de Educación y Desarrollo. Universidad de Guadalajara

Saucedo M. (2016) Investigación de las causas principales de reprobación de los alumnos de Gestión empresarial en el año 2015. Universidad Autónoma del Carmen 
Copyright (c) 2017 Nazaria Martinez Diaz, Laura Elena Urbina Sánchez, David Zepeta Hernández,

Higinio Fernández Sánchez y Erika Mayte Del Ángel Salazar

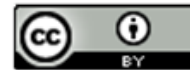

Este tex to está protegi do por una licencia licencia Creative Commons 4.0

Usted es libre para Compartir —copiar y redistribuir el material en cualquier medio o form ato- y Adaptar el documento —remezclar, transformar y crear a partir del material- para cualquier propósito,, incluso para fines comerciales, siempre que cumpla la condición de:

Atribución: Usted debe dar crédito a la obra original de manera adecuada, proporcionar un enlace a la licencia, e indicar si se han realizado cambios. Puede hacerlo en cualquier form a razonable, pero no de form a tal que sugiera que tiene el apoyo del licenciante o 10 recibe por el uso que hace de la obra.

Resumenclelicencia - Textocompletodelalicencia 